\title{
Some Behavioral Considerations on the GPS4GEF Cloud-Based Generator of Evaluation Forms with Automatic Feedback and References to Interactive Support Content
}

\author{
Daniel HOMOCIANU ${ }^{1}$, Dinu AIRINEI ${ }^{2}$ \\ 1"Alexandru Ioan Cuza" University of Iasi, Faculty of Economics and Business \\ Administration, Department of Research \\ ${ }^{2}$ Alexandru Ioan Cuza" University of Iasi, Faculty of Economics and Business \\ Administration, Department of Accounting, Business Information Systems and Statistics \\ daniel.homocianu@feaa.uaic.ro, adinu@uaic.ro
}

The paper introduces some considerations on a previously defined general purpose system used to dynamically generate on-line evaluation forms with automatic feed-back immediately after submitting responses and working with a simple and well-known data source format able to store questions, answers and links to additional support materials in order to increase the productivity of evaluation and assessment. Beyond presenting a short description of the prototype's components and underlining advantages and limitations of using it for any user involved in assessment and evaluation processes, this paper promotes the use of such a system together with a simple technique of generating and referencing interactive support content cited within this paper and defined together with the LIVES4IT approach. This type of content means scenarios having ad-hoc documentation and interactive simulation components useful when emulating concrete examples of working with real world objects, operating with devices or using software applications from any activity field.

Keywords: Short History of Forms, Java Script (JS), Google's Applications, Reasons for using Google Apps Script (GAS), General Purpose System for Generating Evaluation Forms (GPS4GEF), Interactive Support Content for On-Line Forms

$\mathbf{1}_{\mathrm{T}}$ Introduction

The form's history is somehow related to the one of call centers (1965) [1], telephone surveys with typewritten answers, programming languages and web. There are some contemporary studies [2] that even compare the telephone and web-based surveys in terms of reliability. This history was also dependent on the evolution of the programming languages. The last ones evolved and became based on events and graphical user interfaces (GUI) having pictograms, menus, forms with textboxes, radio buttons, check boxes and many other elements. A simple example is Microsoft's Visual Basic (MS VB) [3] that adds some object-oriented features and a GUI to the standard text-oriented BASIC [4]. The MS VB includes forms as core objects starting with the 2.0 version (1992) and it is considered a rapid application development (RAD) [5] tool widely used as a programming environment [6].

The PHP originally named PHP/FI (Personal
Home Page Tools/ Forms Interpreter) was initially used by its developer (Rasmus Lerdorf, 1994) to track the visits of his on-line $\mathrm{CV}$. The later improvements provided interactions with data bases and a framework for developing simple and dynamic web applications [7]. A powerful feature of the PHP is the way it handles HTML forms [8]. The first implementations of forms using the HTML language were in 1995 and 1996. In fact, Dave Raggett had been formalized his new ideas for HTML in a document published as an Internet Draft (March, 1995) containing tables, tabs, footnotes and forms. The developments made in 1996 and 1997 brought the incipient version of what it will be known as HTML 4, offering style sheet and international languages support, as well as extensions to forms and scripting, although is somehow different comparing to the simplicity of the original HTML [9].

The JavaScript (JS) was created by Brendan Eich in 1995 [10] then working at Netscape 
and later at Mozilla. Originally named Mocha it became a standard in 1997 (ECMA262) and evolved to the currently used ECMAScript5 (JavaScript1.8.5 - July 2010). Also part of the AJAX framework architecture (2005) [11] and already well known for its form processing features being among the most sought and used [12] JS's new developments allow us to use it on the server-side or to control user media, open up web sockets for always-on communication, get data on geographical location and device features (accelerometer) [13], and more to come.

In 1996, Larry Page and Sergey Brin have started to collaborate in order to develop a search engine initially named BackRub, tested for a year on Stanford servers and registered late in 1997 as a domain (Google.com) with a name that reminds of the Russian writer Nikolay Vasilyevich Gogol [14] and also of the Edward Kasner's number [15] (Googol) starting with 1 and followed by 100 zeros, suggesting the effort to organize the seemingly infinite amount of information on the Internet. In 2006, Google Docs (originally Writely) and Spreadsheets are lunched as on-line applications. In the same year, Google Apps Premier Edition was lunched in order to offer the cloud computing technology to companies [16]. In 2009, Google Docs gets new features called Drawings [17] and
Forms. The last one is a tool that allows collecting information from users via a personalized survey or quiz. Later, in 2012, it was integrated in Google Drive - a service based on the Google File System [18] through which we can create, send, keep and work in collaboration to our files (videos, photos, docs, pdfs), all in one place.

\section{Why using GAS behind the GPS4GEF Prototype}

GAS is defined by Google as a JS cloud scripting language that provides easy ways to automate tasks across Google products and third party services and build web applications [19]. Google Forms, Spreadsheets and Sites also incorporate GAS to write code within documents in a similar way to Visual Basic for Applications (VBA) in Microsoft Office. The scripts can be activated either by user action or by a trigger in response to an event [20]. Therefore we consider that GAS is better than VBA in terms of goals although it lacks the transparency and speed of VBA in terms of error correction. The fig. 1 depicts an example of delay of more than 12 hours when sending the full error description in order to debug a GAS script that ends with failure (we have got no e-mail feedbacks as authors).

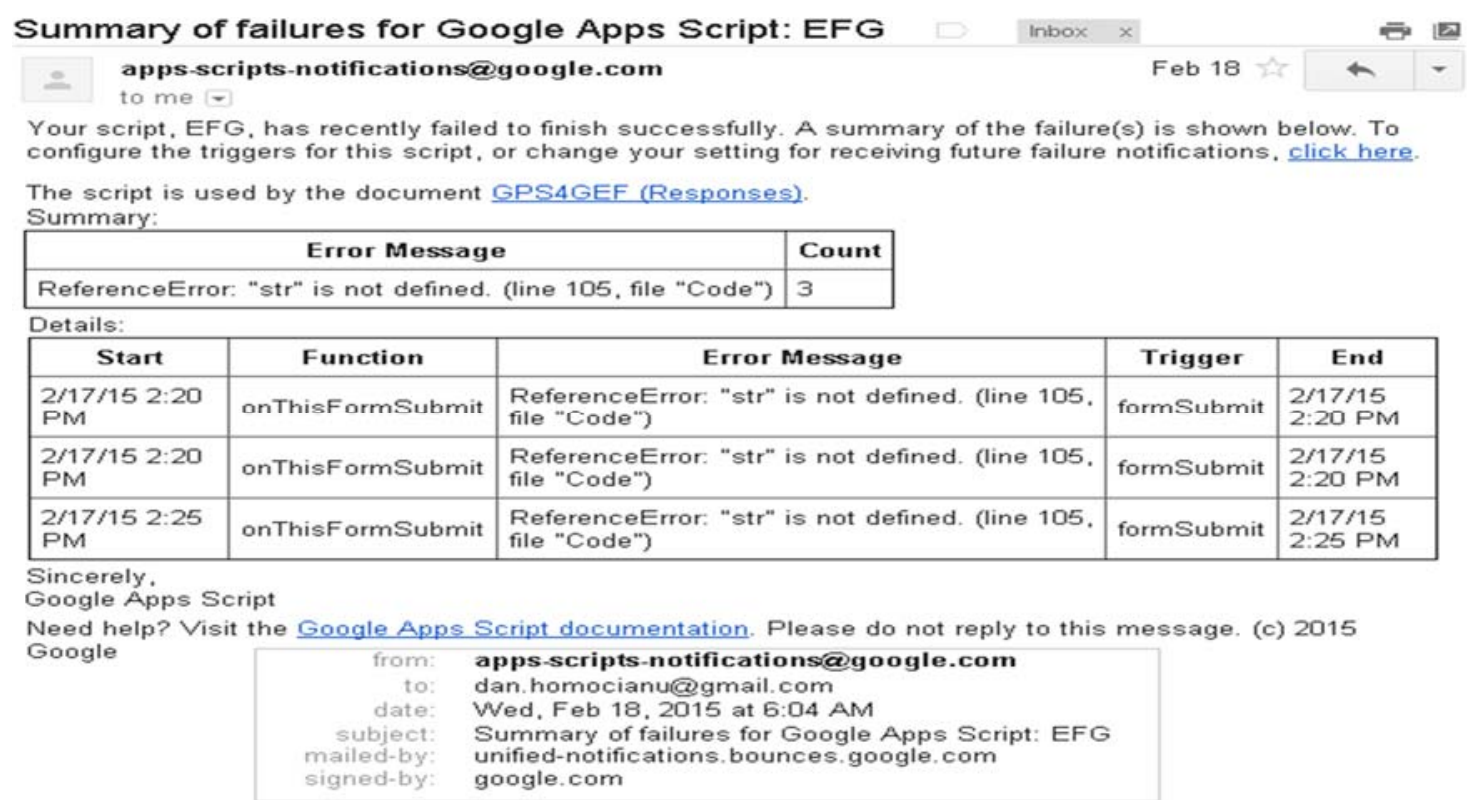

Fig. 1. Programming the GPS4GEF form generator - example of hardly acceptable delay in transmitting the full error description after a GAS script failure 
There are many on-line available examples [21] proving GAS's ability to interact with data sources (Sheets [22] for single tables and Google Cloud SQL [23] for databases [24]) and to automatically generate results [25]. These examples coupled with the similarity between JS [26] and GAS [27] somehow compensate the previously mentioned lacks in real-time reporting of code errors (fig.1). This is why we have accepted to build up the GP4GEF prototype by using GAS. Instead of working with many tables in databases (e.g. Categories, Questions, Answers and so on - Figure 2), we have chosen to test GAS with Google Sheets registers (44 characters id each - Figure 3) with many sheets (categories) and many lines (ques- tions) in one sheet. Every single line will store data about a question in simple afferent fields but also about all the answers and all the correct ones in two complex fields with text sequences separated by a comma and a space (default Google separation when transmitting many form options to a collector spreadsheet) and all these without losing contact to the relational model. The conversion from this one to Google Sheets as used by Google Forms [28] (above) was done after testing some Access Crosstab queries reminding of OLAP [29] and followed by exports to Excel (Figure 2). A way back (static multidimensional data to tuples) by using Google Refine is described in our previous paper [30] written and published in 2014.

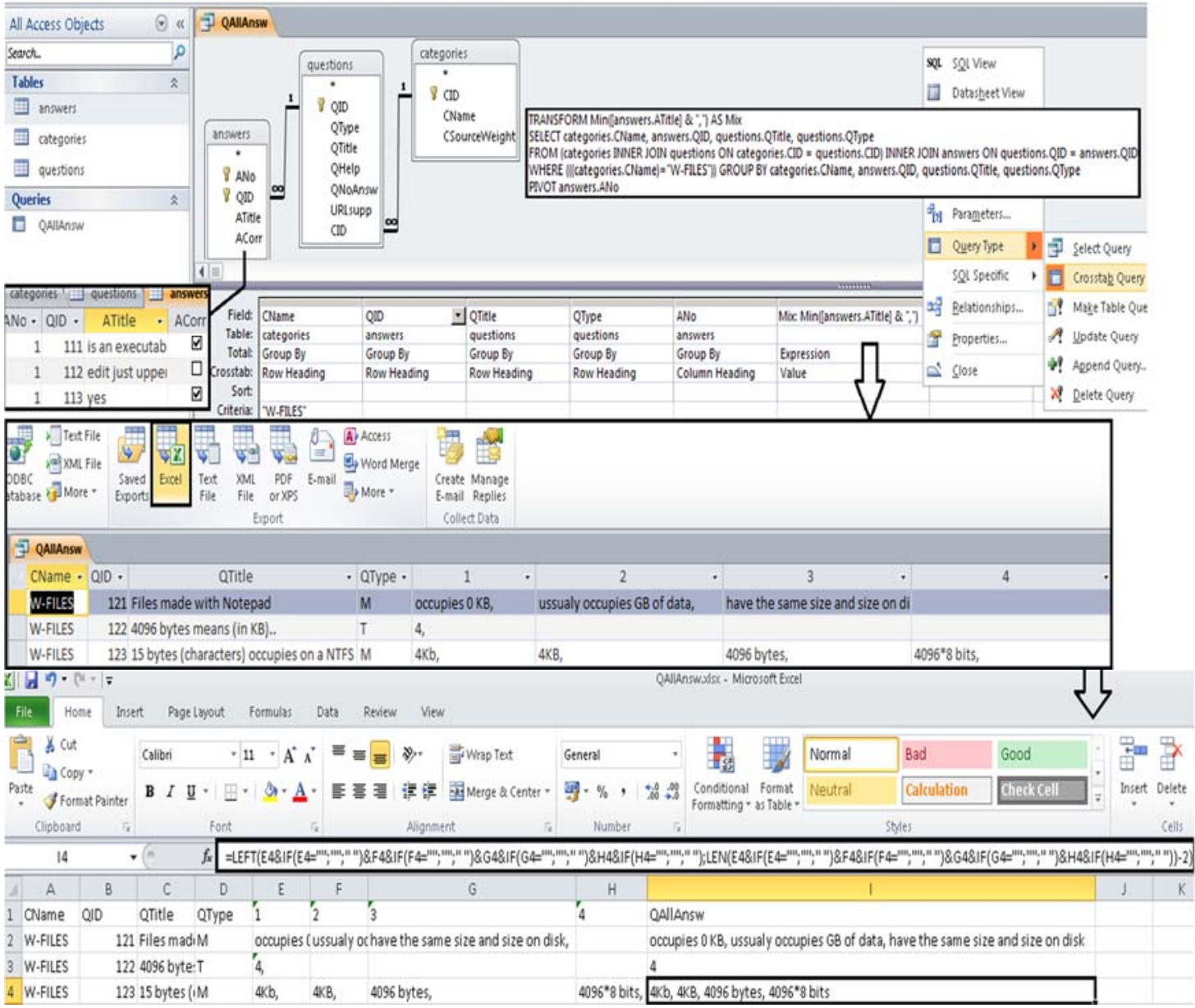

Fig. 2. Using GPS4GEF as instructor - example of query based conversion from an already existent relational database (Microsoft Access) to the GPS4GEF's source format

Following the pattern above (Figure 2) for each category, an instructor should end integrating the results in just one spreadsheet file with many sheets for categories as required for a GPS4GEF's data source (upper left of Figure 3) in a short amount of time (minutes 
to hours). The next step to follow in order to get something from the GPS4GEF form generator is to share the data source with the Google account associated to this form generator (owner of the application and coauthor of this paper - bottom of Figure 3) or to another new but similar form generator. In this context, similar means in our opinion a form generator with a corresponding collector having the same source code as the one presented below (those 114 bold lines after the Figure 4) and the same triggers applied on it (Figure 5). However any author should feel free to use/change these code lines and associate the resulting application to his own Google account.

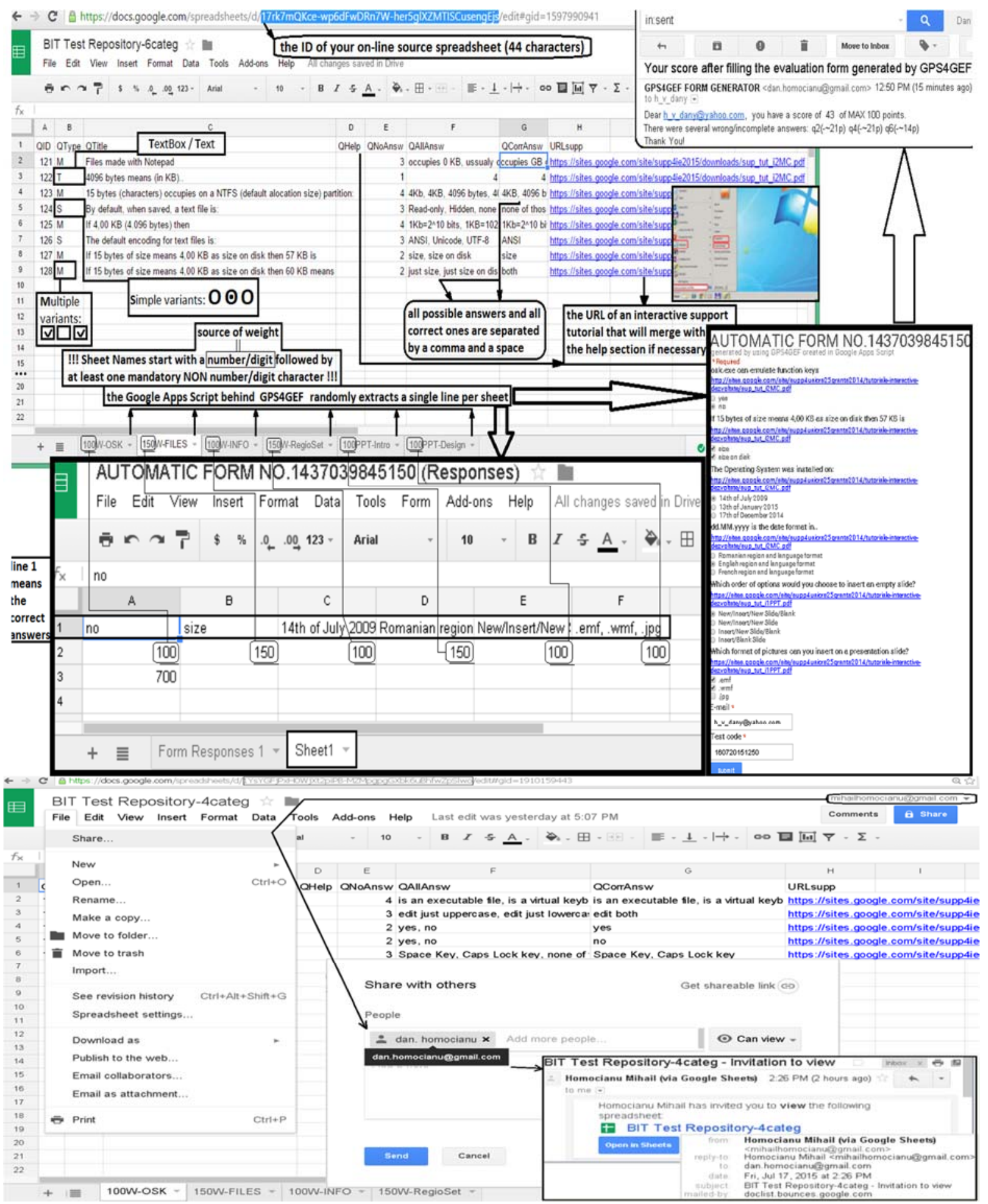

Fig. 3. Using GPS4GEF as instructor - preparing and sharing a source for this generator and how that is influencing the generated form $\&$ its corresponding data collector spreadsheet 
C 1 i https://docs.google.com/forms/d/1c8MB5v8P-rtiCueFimScjB1H3cPs8cynJXIpRx_Yn0s/edit

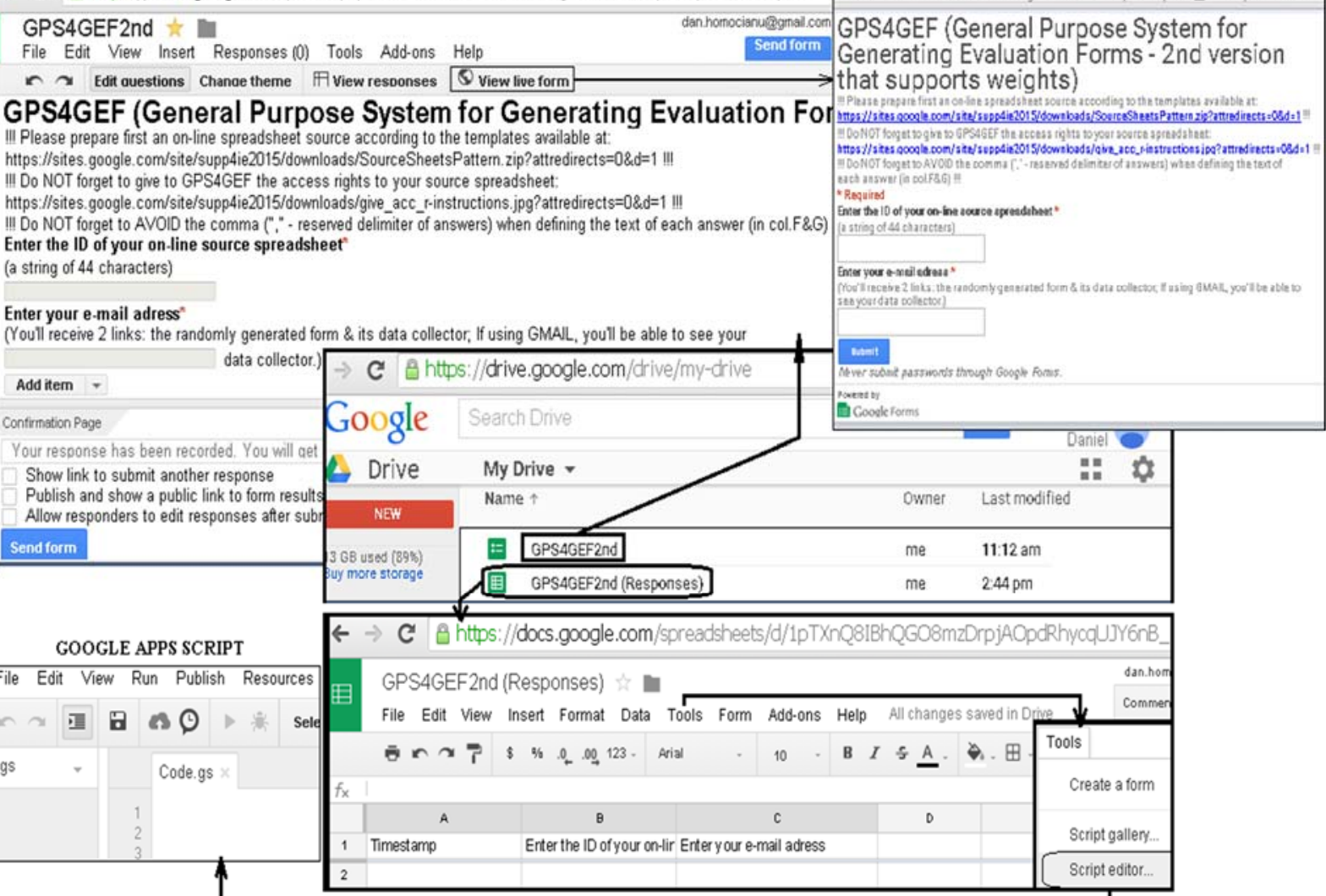

Fig. 4. Designing GPS4GEF's form (2 textboxes) and source code [31]

(behind its data collector)

Source code: GPS4GEF form generator as onThisFormSubmit [32] \& other 11 functions [33]

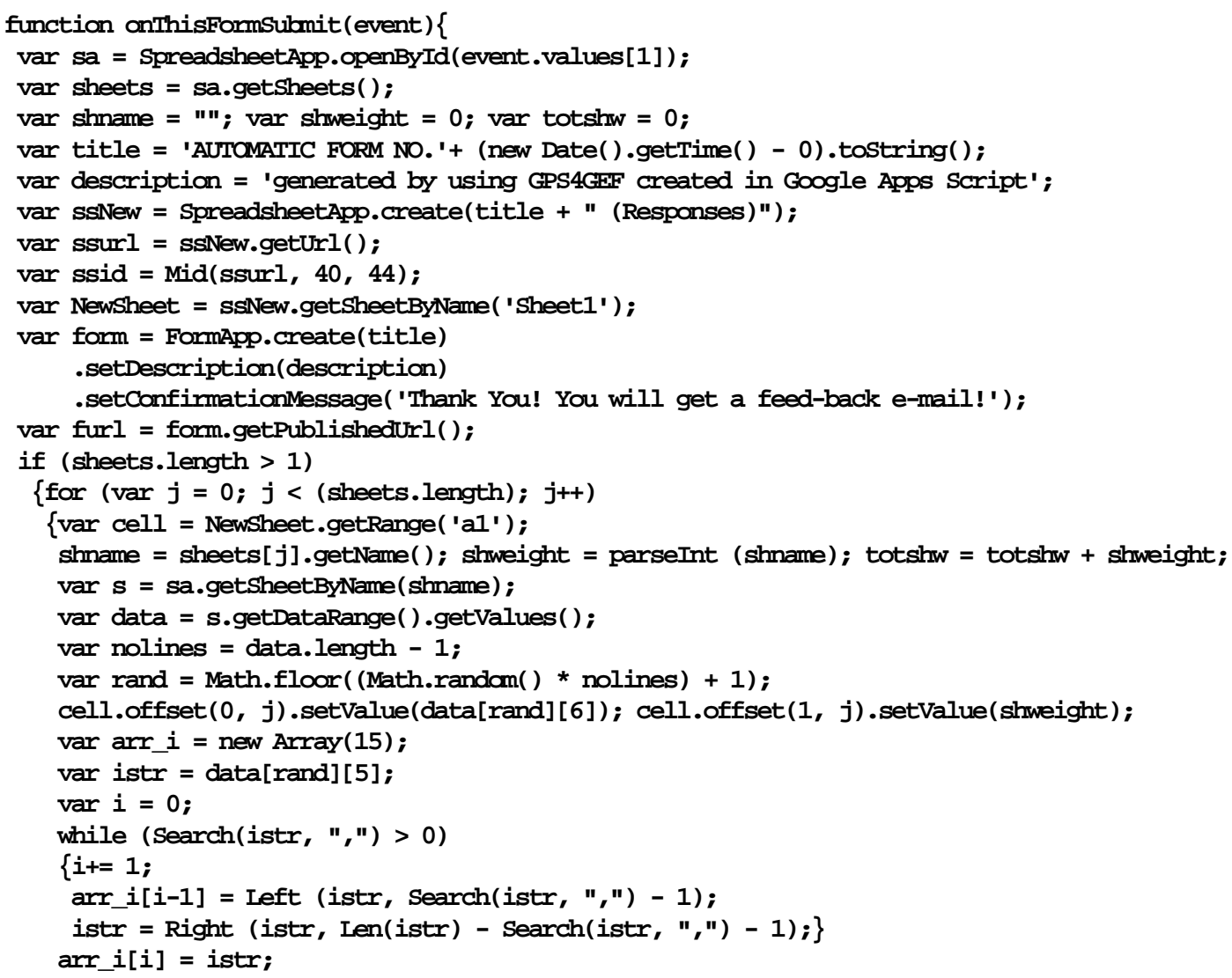




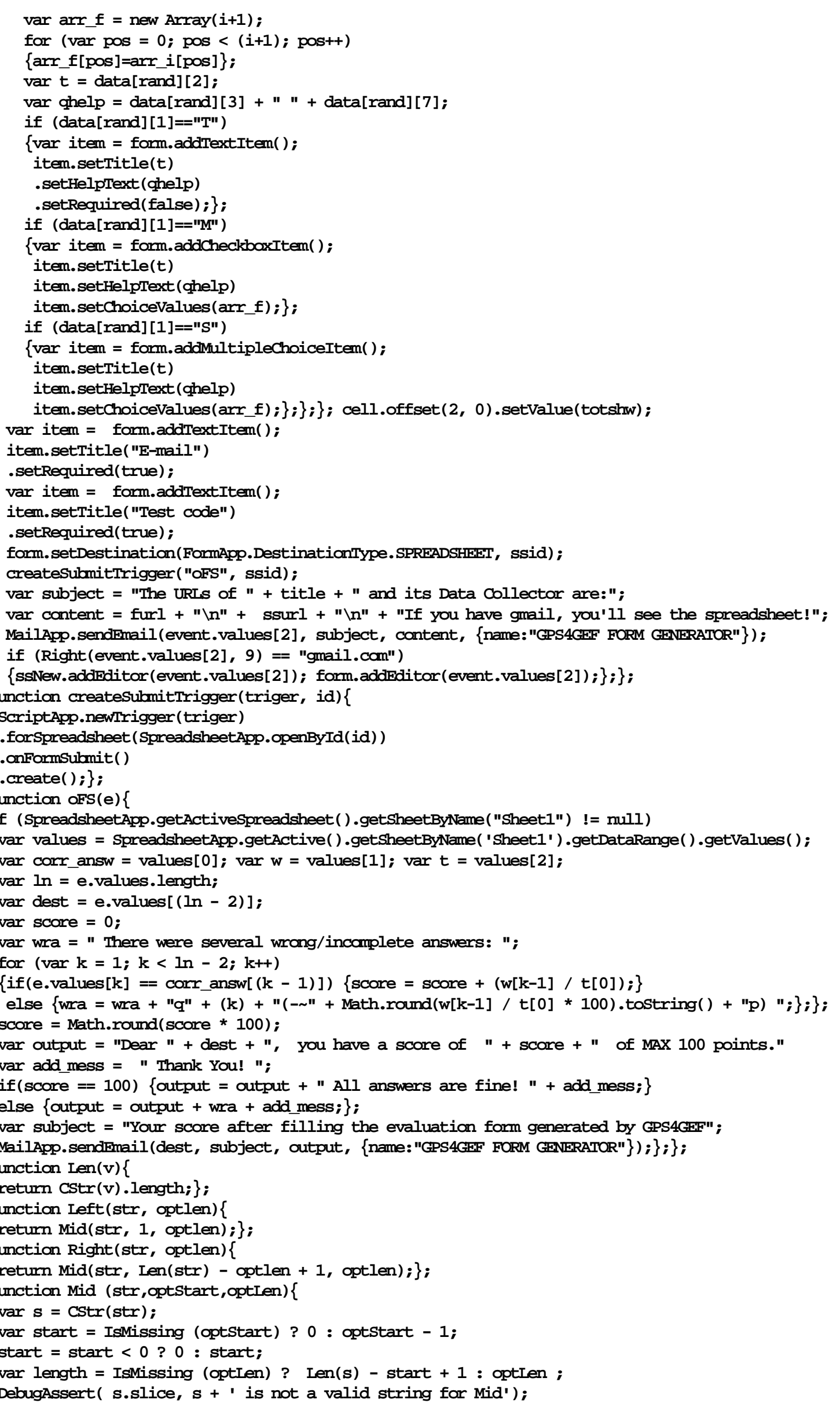




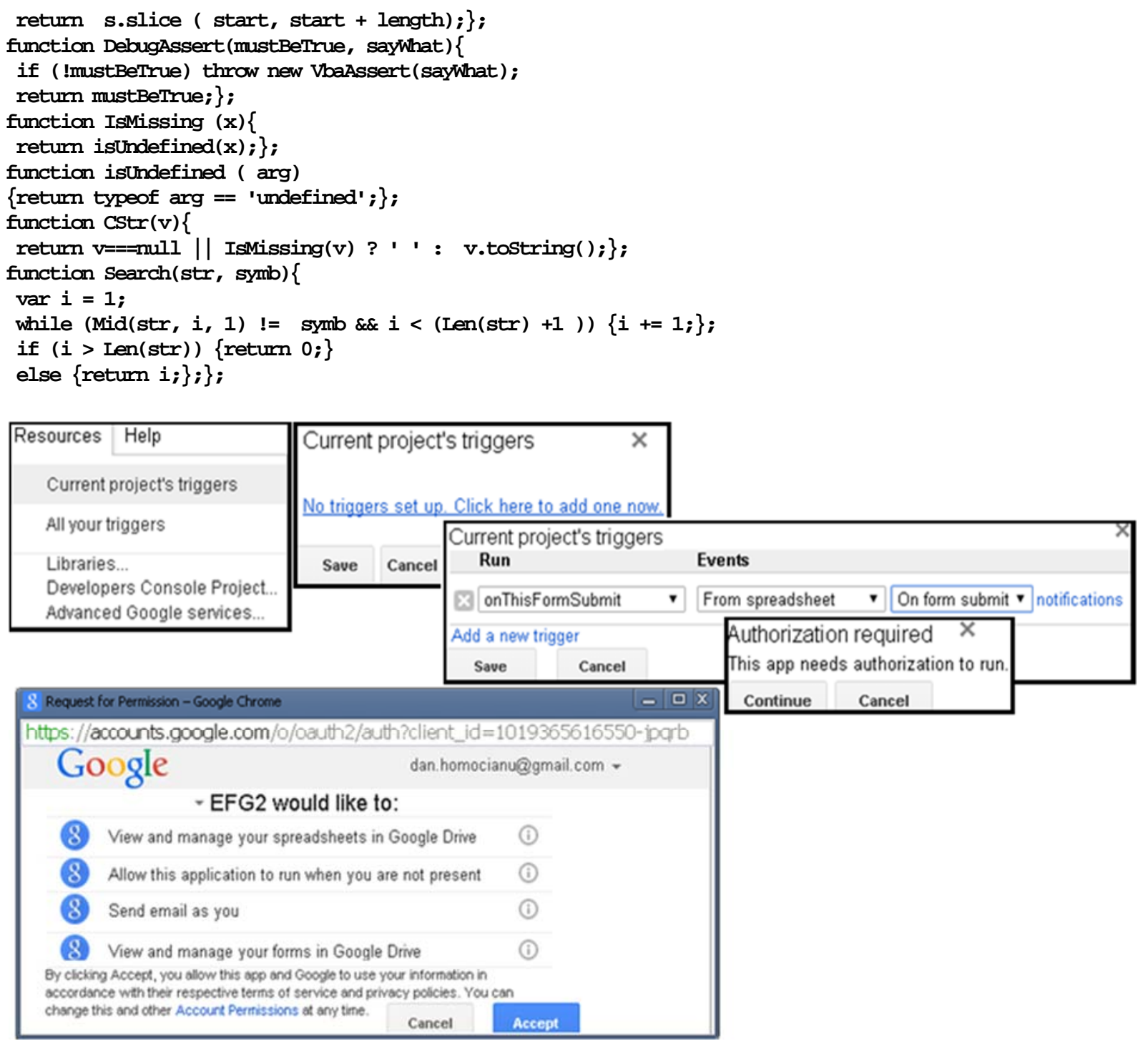

Fig. 5. Programming the GPS4GEF's behavior - how to trigger the main function defined in GAS (onThisFormSubmit) to the GPS4GEF's form submit event (source ID \&author's email)

The GPS4GEF's source format is based on spreadsheets because of a few reasons: it is the native format used by Google Forms to store responses; it is an intuitive well connected format [34] many users involved in assessment activities have experience with rather than .XML, .JSON [35]; the Google Fusion Tables [36] complex solution [37] for cloud databases is still experimental [38]. This model could also be used when trying to extract more than one question per category. This can be done by duplicating a category (copy and paste of a sheet with a name containing a number used following the decisional utility [39] method) although it means a certain risk of duplicating a question in the final generated evaluation form, with the possibility of correction by editing (Figure 6) in two simple steps [40] corresponding to the resulting form and its data collector.

For the development part, the most important piece of code is represented by a function named onThisFormSubmit that has many roles: it reads the author's data source; it builds a form and its corresponding two sheets data collector (Sheet1 in Figure 3 correct responses \& weights) after reading one random question per category (sheet) and accessing all the sheets of the source; it generates and transmits two corresponding URLs to the author that has editing rights on both objects; it dynamically builds an evaluation trigger (oFS - Figure 9) suited for the generated form (\& its collector) that will compute 
and send (Figure 7) a score after a person fill it.

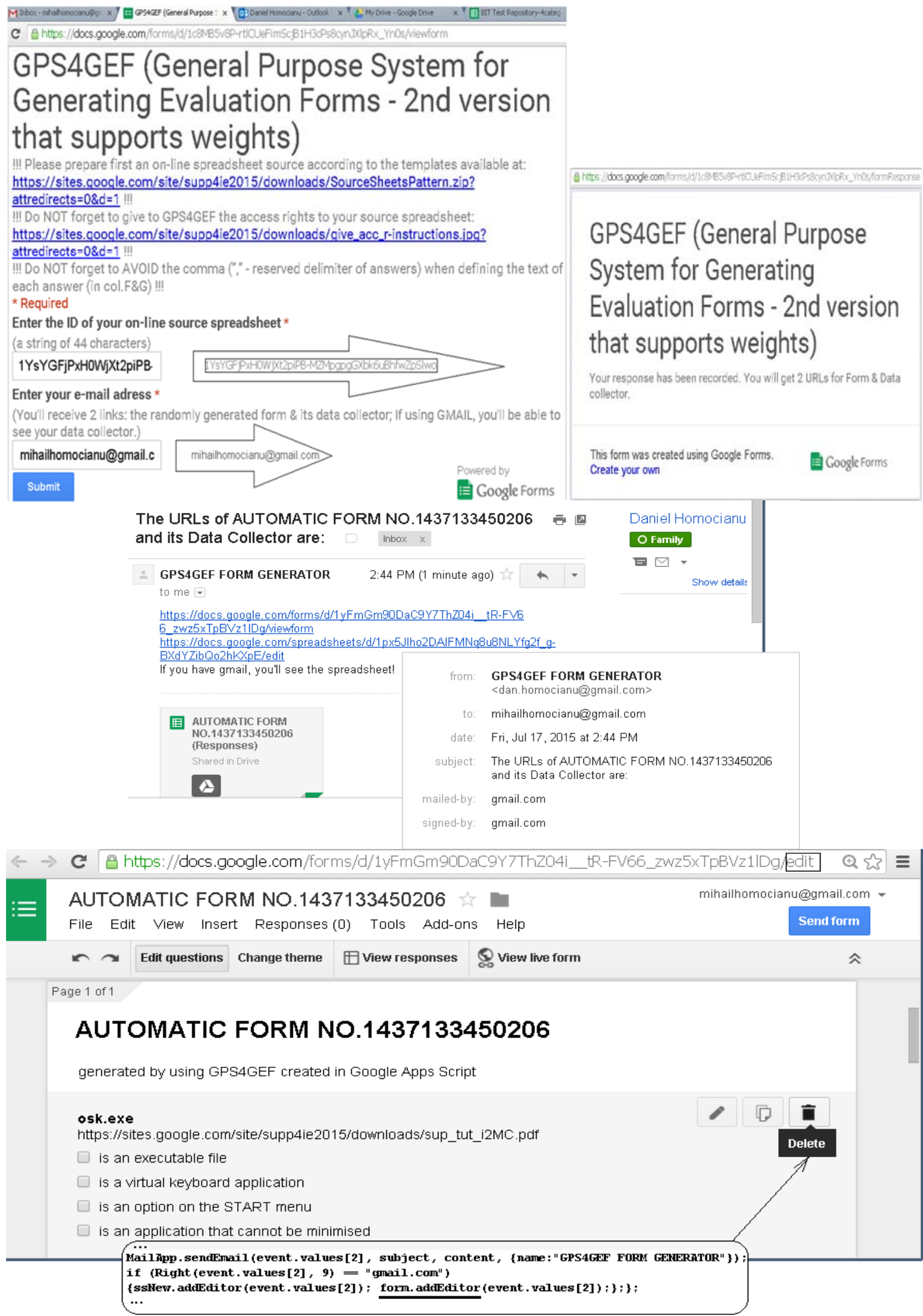

Fig. 6. Using the GPS4GEF [41] as instructor - automatically generating an evaluation form and getting the corresponding URLs that grant editing access (both form and its spreadsheet) 


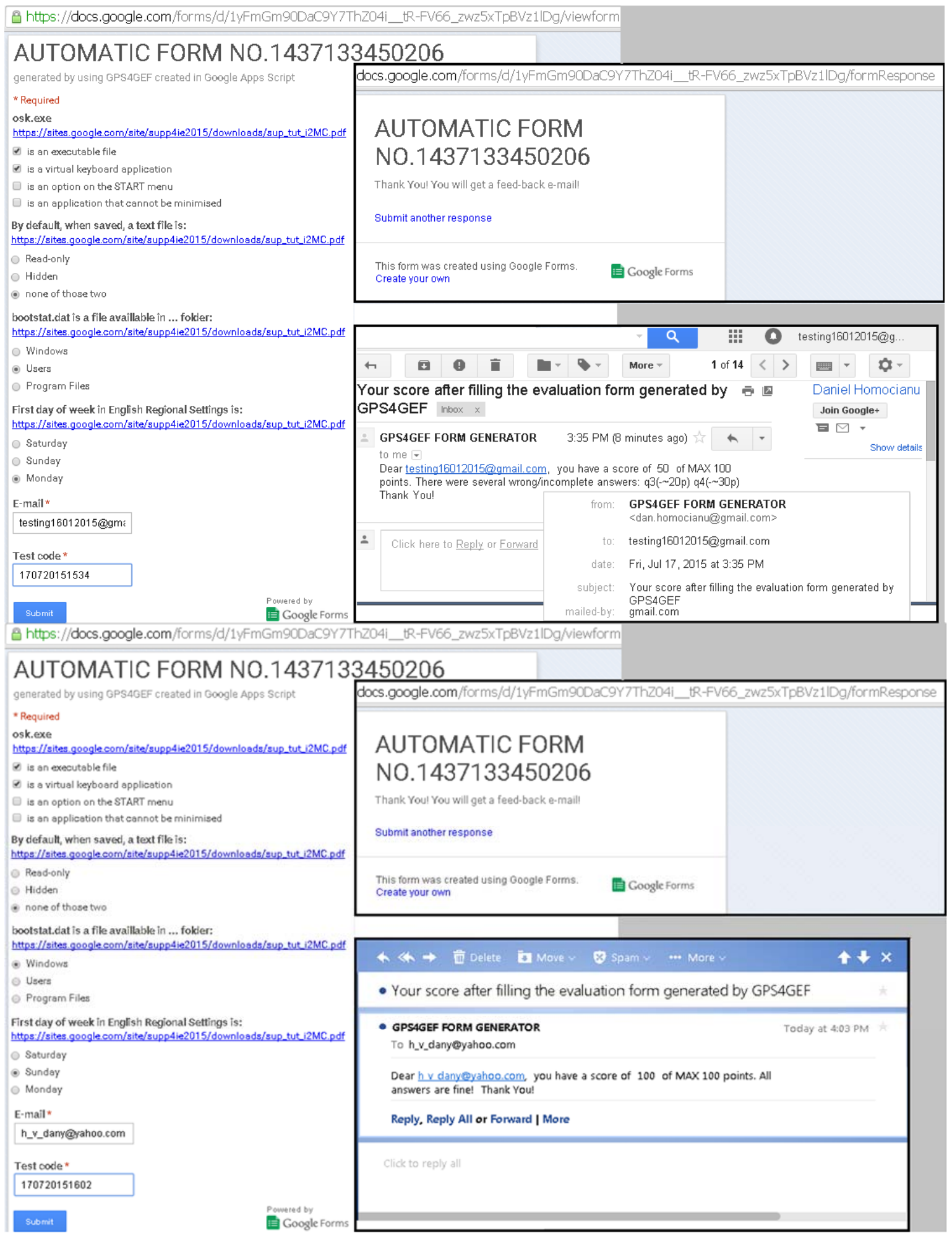

Fig. 7. Using the GPS4GEF as an evaluated person - filling the evaluation form and automatically getting the corresponding feed-back

The great advantage of this architecture is represented by the fact that any author / instructor have enough control (edit rights Figures.6, 8) on the content associated to both URLs. Asked how the idea of GPS4GEF came, we admitted that the first call was to adapt and associate a piece of GAS script asynchronously accessing the corresponding source to every manually designed Google form [42], with many limitations and technical requirements for final users. 


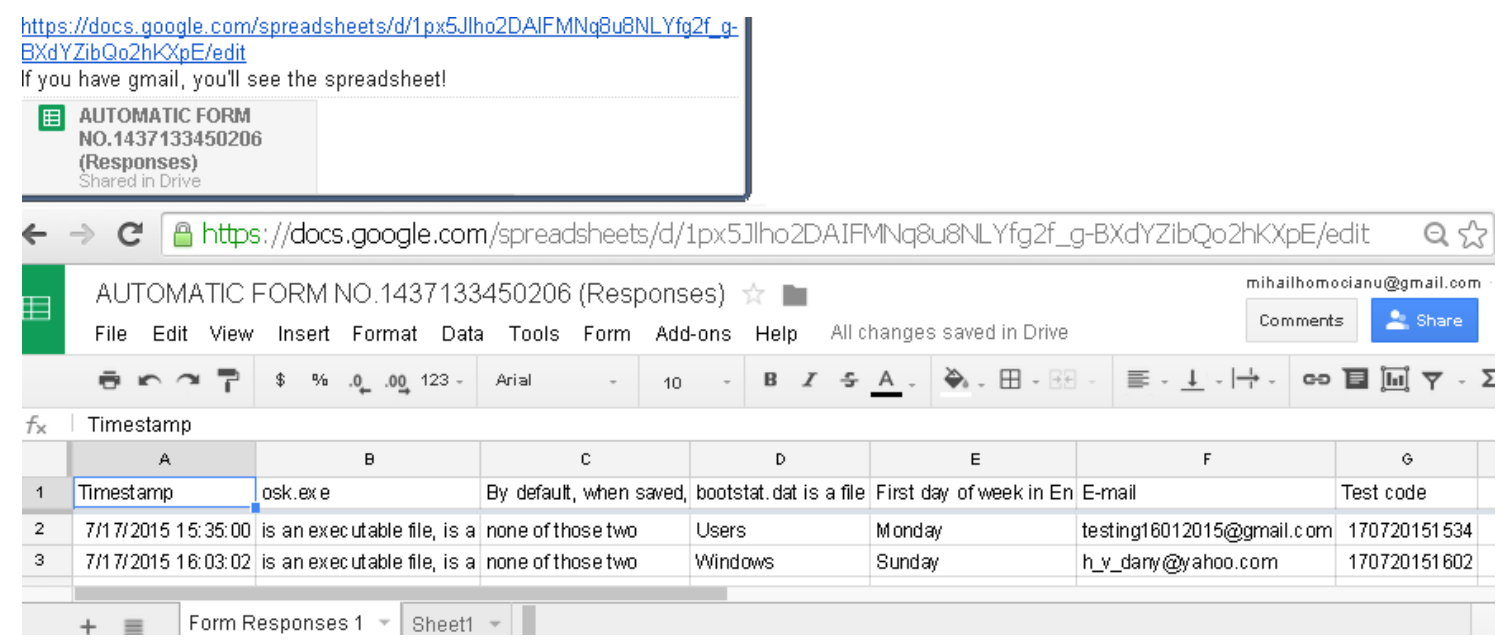

Fig. 8. Using the GPS4GEF as instructor - accessing detailed information about the answers

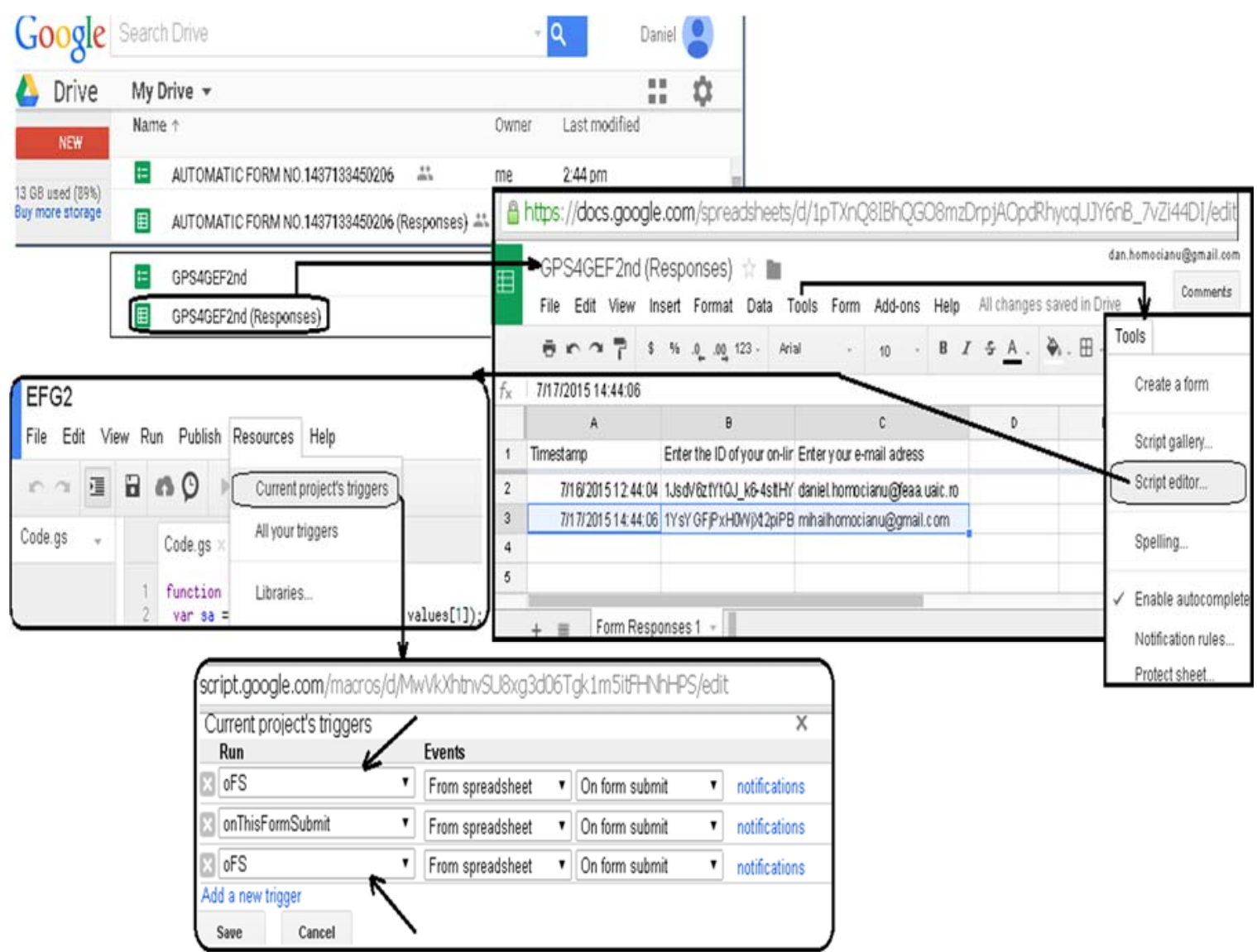

Fig. 9. Checking the GPS4GEF's triggers - observing the dynamically generated ones (oFS) meaning two evaluation forms successfully generated and having post-submit behavior

Some additional restrictions still to be set behind the form generator (onThisFormSubmit function) are those required in order to eliminate the possibility of an evaluated person to complete the same form many times. Moreover the message sent to an evaluated person should contain a reference to the form's title (unique id) and URL because of speed of search reasons on the e-mail subject. That should be done as resumed in Figure 10. Another improvement to do is to automatically send to authors the $3^{\text {rd }}$ URL ( $2^{\text {nd }}$ link to the form but in edit mode) relieving them from manually changing the "view" part of URL to "edit" (Figure 6). 


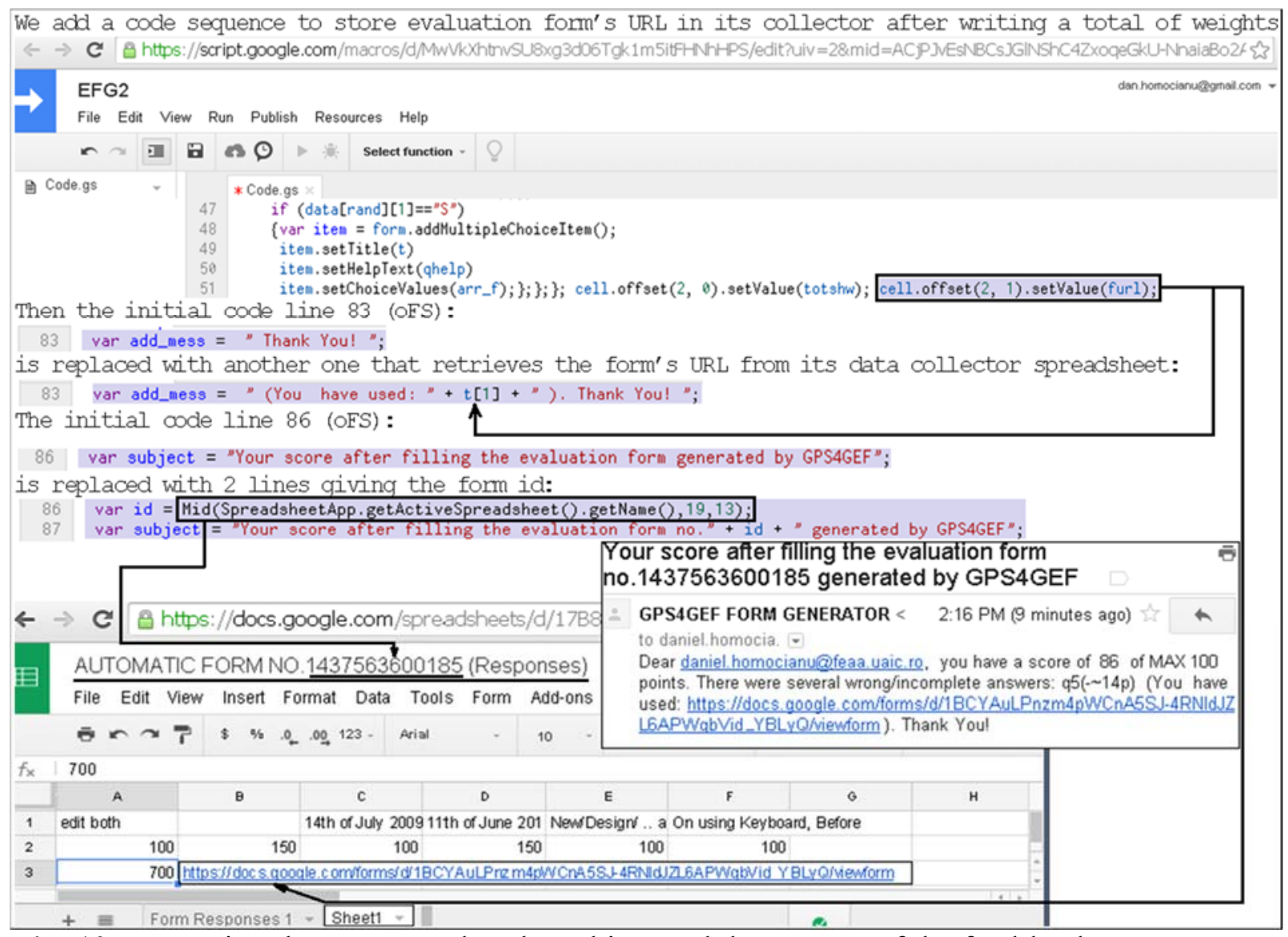

Fig. 10. Improving the source code - the subject and the content of the feed-back message are now sent to the evaluated person with the form's ID and URL

The chosen name (GPS4GEF - General Purpose System for Generating Evaluation Forms) starts with a $\mathrm{G}$ and contains two such symbolic characters as an obvious tribute to the Google technology used to develop this prototype and suggests even a new direction (GPS) to follow in order to increase the productivity of evaluation and assessment tools available for any instructor, teacher, professor or educational researcher (code name Jeff / GEF).

\section{Conclusions}

The general conclusion of this paper highlights the context of knowledge, education and lifelong learning involving a special attention to assessment and evaluation activities enhanced by instruments capable to move the focus from technical and implementation details to patterns to follow in order to increase the productivity.

The examples reveal the components of this functional system implemented by using mostly free technologies available for any internet user. The same examples suggest desired features on transparency, availability, flexibility, and speed of data extraction, processing and reporting, as key performance issues for any activities involving evaluation and assessment.

In order to contribute to the full understanding of the GPS4GEF's architecture and behavior we have tried our best in putting together a lot of documentation and support references including indirect links to video tutorials, the source code and a lot of complex captures based on a cause-effect approach and following the old saying ,a picture paints a thousand words".

\section{Acknowledgment}

This paper was funded by the Department of Research of the Faculty of Economics and Business Administration from the "Alexandru Ioan Cuza" University of Iasi. 


\section{References}

[1] www.callcentrehelper.com/the-history-ofthe-call-centre-15085.htm

[2] K. Braunsberger, H. Wybenga and R. Gates, "A comparison of reliability between telephone and web-based surveys", Journal of Business Research, 60(7), pp.758-764, July 2007

[3] whatis.techtarget.com/definition/BASIC Beginners-All-purpose-SymbolicInstruction-Code

[4] www.z80.eu/basic.html

[5] V. Himpe, Visual Basic for Electronics Engineering Applications. Dorchester: Elektor Electronics Publishing, 2009, pp.33

[6] www.max-visual-basic.com/history-ofvisual-basic.html

[7] php.net/manual/en/history.php.php

[8] php.net/manual/en/tutorial.forms.php

[9] www.w3.org/People/Raggett/book4/ ch02.html

[10] www.w3schools.com/js/js intro.asp

[11] D. Homocianu, "AJAX or the New Standard for Faster Web Interfaces", in Proc. The International Workshop on Economic Theory and Practice in Knowledge Society, Iasi, Romania, 2007, pp.302, ssrn.com/abstract $=2382557$

[12] www.javaworld.com/article/2077176/ scripting-jvm-languages/using-javascriptand-forms.html

[13] www.w3.org/community/webed/wiki/ A_Short_History_of_JavaScript

[14] www.britannica.com/biography/Nikolay -Gogol

[15] dictionary.reference.com/browse/googol

[16] www.google.com/about/company/ history/\#top

[17] readwrite.com $/ 2009 / 03 / 25 /$ google_docs inserts drawings

[18] C. Boja, A. Pocovnicu and L. Bătăgan (2013). Distributed Parallel Architecture for "Big Data". Informatica Economica Journal [Online]. 2(16). Available: revistaie.ase.ro/content/ 62/12\%20\%20Boja.pdf

[19] www.google.com/script/start

[20] self.gutenberg.org/articles/google_docs

[21] www.synergyse.com/blog/top-10- google-apps-scripts-for-education

[22] stackoverflow.com/search? $\mathrm{q}=$ google + sheets

[23] cloud.google.com/sq1/docs/introduction

[24] ctrlq.org/code/19978-connect-to-googlecloud-sql-from-apps-script

[25] stackoverflow.com/questions/tagged/ google-apps-script

[26] www.w3schools.com/jsref/tryit.asp? filename $=$ tryjsref_random 3

[27] blog.ditoweb.com/2013/10/choosingbetween-google-apps-script-and.html

[28] stackoverflow.com/questions/26393556/ how-to-prefill-google-form-checkboxes

[29] D. Airinei and D. Homocianu, "Data Visualization in Business Intelligence", in Proc. The WSEAS MCBEC International Conference, Iasi, Romania, 2010, pp.164, ssrn.com/abstract $=2381812$

[30] D. Homocianu and D. Airinei, "Consolidating Source Data in Audit Reports", Audit

Financiar Journal, 116(8), pp.15, August 2014, ssrn.com/abstract $=2502539$

[31] D. Homocianu and D. Airinei, "General Purpose System for Generating Evaluation Forms (GPS4GEF)", in Proc. The 14th International Conference on Informatics in Economy, Bucharest, Romania, 2015, www.conferenceie.ase.ro, ssrn.com/abstract $=2602708$

[32] sites.google.com/site/supp4ie2015/ downloads/OTFS.tiff?attredirects $=0 \& \mathrm{~d}=$ 1 - original commented code

[33] sites.google.com/site/supp4ie2015/ downloads/OFS_other_f.tiff?attredirects= $0 \& \mathrm{~d}=1-$ original commented code

[34] D. Homocianu, Decision Support Systems in the Context of Knowledge Society, "Alexandru Ioan Cuza" University Publishing Iasi, 2009, pp.121, ssrn.com/abstract $=2384380$

[35] O. Dospinescu and M. Perca (2013). Web Services in Mobile Applications. Informatica

Economica Journal [Online]. 2(17). Available: revistaie.ase.ro/content/ 66/02\%20-\%20Dospinescu,Perca.pdf

[36] susanemcgregor.com/using-googlefusion-tables-as-a-web-application- 
database

[37] stackoverflow.com/questions/12949701 /use-fusion-table-like-a-database-withphp-and-how-to-dismiss-the-promptpage-whe

[38] support.google.com/fusiontables/answer $/ 2475373$ ?hl=en

[39] D. Airinei and D. Homocianu (2009). The Geographical Dimension of DSS Applications. Scientific Annals of Alexandru Ioan Cuza University of Iasi [Online]. Tome LVI. Available: anale.feaa.uaic.ro/anale/resurse/49 I01 Airi nei.pdf

[40] D. Homocianu, D. Airinei and F. Du- mitriu, "2CASE - Cloud Computing Based Audit Staff Evaluation by Using the GPS4GEF On-Line Prototype", Audit Financiar Journal, 7(127), pp.105, July 2015, ssrn.com/abstract $=2622926 \quad$ [41] docs.google.com/forms/d/1c8MB5v8PrtlCUeFimScjB1H3cPs8cynJXlpRx_Yn0

[42] D. Homocianu, "The LIVES4IT Approach on Access to Documentation Resources, Education, Training and Research", in Proc. The 6th International Conference on Information Science and Information Literacy, Sibiu, Romania, 2015, bcu.ulbsibiu.ro/conference/ proceed.htm, $\quad$ ssrn.com/abstract $=2602711$

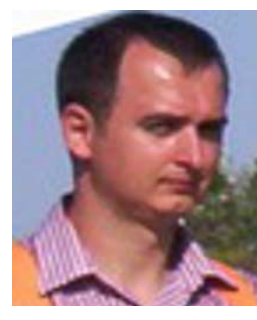

Daniel HOMOCIANU has graduated the Faculty of Economics and Business Administration (FEAA) - Alexandru Ioan Cuza University of Iasi (UAIC) in 2002. He holds a PhD diploma in Cybernetics and Economic Statistics from 2009 and he is currently a researcher within the Department of Research of FEAA UAIC. He is author/co-author of 8 books and 57 scientific articles in journals and proceedings of international conferences in the field of Business Information Systems.

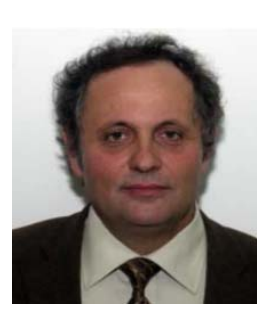

Dinu AIRINEI has graduated the Faculty of Economic Sciences from UAIC in 1978. He holds a PhD diploma in Economics from 1997 and he had gone through all didactic positions since 1982 when he joined the staff of the Faculty of Economic Sciences, teaching assistant in 1982, senior lecturer in 1990, associate professor in 1998 and professor in 2000. Currently he is professor of Business Information Systems within the Department of Accounting, Business Information Systems and Statistics at FEAA UAIC. He is the author of more than 22 books and over 75 journal articles in the field of Business Information Systems, Decision Support Systems, Data Warehouses and Business Intelligence. 